\title{
Comparação entre difração de raios X e "método do furo cego" para medição de tensões residuais em barras cilíndricas
}

\author{
ROCHA, A.S. ; NUNES, R.M. ${ }^{\text {I. }}$ HIRSCH, T.K. ${ }^{\text {II }}$ \\ ILaboratório de Conformação Mecânica - LdTM - UFRGS - Centro de Tecnologia , Rio Grande do Sul, RS. \\ e-mail: alexandre.rocha@ufrgs.br, rafael.nunes@ufrgs.br \\ II IWT - Stiftung Institut für Werkstofftechnik - Bremen, Alemanha \\ e-mail: hirsch@iwt-bremen.de
}

\section{RESUMO}

As tensões residuais na superfície e subsuperfície de componentes mecânicos, durante sua manufatura e em serviço, podem afetar o desempenho e representar um importante potencial aos seus desvios de forma e dimensão. Estas tensões residuais também representam um dos principais potenciais para distorções (isto é, empenamentos e mudanças dimensionais) em componentes mecânicos durante a manufatura. Por isso são de grande importância a determinação da distribuição e o controle destas tensões residuais em cada etapa do processo de manufatura. Neste trabalho, foram realizadas comparações entre os resultados de medições de tensões residuais pelos métodos de difração de raios X (XRD) e Furo cego (Holedrilling). Foram realizadas análises da variação das tensões residuais decorrentes do processo de fabricação de barras cilíndricas de aço AISI 1045. As barras foram analisadas com diferentes níveis de tensões residuais. A partir dos valores de deformações obtidos, foram calculadas as tensões residuais na direção longitudinal para cada ponto ensaiado. Com este trabalho, foi obtida uma comparação do desenvolvimento de tensões residuais na região superficial até uma profundidade de $1 \mathrm{~mm}$ de barras cilíndricas.

Palavras-chaves: Tensões Residuais, difração de raios X, método do furo cego.

\section{Comparison between $\mathrm{X}$-ray diffraction and hole-drilling for measuring residual stresses in cylindrical bars}

\section{ABSTRACT}

The residual stresses in the surface and subsurface of mechanical components during the manufacturing process can affect the behavior and represent a significant potential for deviations in shape and size. These residual stresses also represent one of the main potentials for the distortion (which are bending and dimensional changes) in mechanical components during the manufacture. Therefore, the determination of the distribution and the control of these residual stresses in each stage of the manufacturing process is of great importance. In this work, analysis of the variation of the residual stresses were accomplished in cold-drawed bars of AISI 1045 steel due to the manufacturing process. The bars were analyzed by X-ray diffraction (XRD) and hole-drilling (H.D) to characterize the residual stresses. Starting from the values of strains obtained, the longitudinal residual stresses were calculated for each tested point. With this work, a detailed view of the development of stress in the surface to a depth of $1 \mathrm{~mm}$ of bars after the process steps was obtained.

Keywords: Residual stress, X-ray diffraction, hole-drilling.

\section{INTRODUÇÃO}

O controle de qualidade durante os processos de manufatura de componentes mecânicos é de grande importância para a obtenção de um produto final com as características desejadas. Um dos principais problemas enfrentados pela indústria metal-mecânica são os desvios de forma e dimensão de componentes durante os processos de manufatura. Estas distorções que estão associadas ao nível e distribuição das tensões residuais no componente fabricado. A medição, controle ou monitoramento de tensões residuais durante toda rota de processamento do material é de vital importância para assegurar os desvios de forma e dimensão previstos no projeto de Engenharia em etapas subseqüentes de processamento do material. 
Tensões residuais são as tensões existentes em um corpo sólido sem aplicação de forças externas (incluindo gravidade) ou gradientes térmicos. Todo sistema de tensões residuais está em equilíbrio e o somatório das forças resultantes e dos momentos produzidos é zero. O valor máximo em módulo que as tensões residuais poderão chegar é o próprio limite de escoamento do material [1].

Existem várias técnicas de medição de tensões residuais, cada uma apresentando suas vantagens e limitações. A determinação das tensões residuais em um componente muitas vezes pode ser realizada com a utilização de mais de uma técnica de medição. Desta forma, utilizam-se duas ou mais técnicas conforme as características do componente que será medido. Segundo Lu [1]], os principais parâmetros que devem ser levados na escolha da técnica de medição, os principais parâmetros são: A natureza do componente, tipo de tensões residuais, gradiente de tensões residuais, geometria do componente e o custo final da medição.

Neste trabalho objetivou-se comparar as técnicas de difração de raios X e hole-drilling para a medição de tensões residuais em barras cilíndricas de aço AISI 1045.

A produção de barras cilíndricas do aço AISI 1045 atendendo determinados requisitos de resistência mecânica e geometria pode ser feita a partir de rolos de fio-máquina, que são endireitados e trefilados num processo conhecido como trefilação combinada.

O fio máquina é obtido em um processo de laminação a quente, após o fio-máquina é bobinado em rolos e estocado. Antes do processo de trefilação o fio-máquina é decapado com solução ácida para remoção de carepa e posteriormente neutralizada com solução alcalina. O processo de manufatura inicia pelo préendireitamento do fio-máquina que é fornecido em bobinas, sem este pré-endireitamento o processo de trefilação ficaria inviável. A próxima etapa é o jateamento, que tem como objetivos a remoção de carepas provenientes do processo de laminação e consequentemente a limpeza da superfície. Após o jateamento é feita a trefilação e em seguida a operação de corte da barra (6 metros). Após o corte, ainda são necessários um polimento e endireitamento final, conhecido como "PERC".

Esse processo impõe deformações plásticas ao material que induzem distribuições de tensões residuais, as quais se alteram durante as diferentes etapas do processo. Este artigo apresenta uma comparação das medidas obtidas utilizando-se as duas técnicas de medição de tensões residuais para barras cilíndricas produzidas pelo processo de trefilação combinada.

Um aspecto importante associado às tensões residuais é a distorção, que em barras longas se manifesta principalmente por um empenamento. Em etapas subseqüentes de manufatura, tais como tratamentos térmicos e operações de usinagem, as tensões residuais vão sofrendo redistribuições durante toda a rota de fabricação. As tensões residuais podem ser aliviadas (ou intensificadas) em etapas subseqüentes de processamento do material, fora da usina, tais como, usinagem e tratamentos térmicos, resultando em distorções indesejadas.

\section{MATERIAIS E MÉTODOS}

As amostras analisadas foram retiradas de quatro diferentes etapas do processo de manufatura, sendo elas: pré-endireitamento e jateamento do fio-máquina ( $1^{\mathrm{a}}$ etapa), trefilação ( ${ }^{\mathrm{a}}$ etapa) e polimento e endireitamento por rolos cruzados - "PERC” ( $3^{\text {a }}$ etapa). Os diâmetros das amostras são de 21,65mm para o fio-máquina ( $1^{\text {a }}$ etapa) e 20,25mm para as barras ( $2^{\mathrm{a}}$ e $3^{\mathrm{a}}$ etapas). Na obtenção de amostras foi levada em conta a orientação das amostras retiradas em relação a um referencial fixo, esta orientação é importante para a comparação entre as técnicas de medição devido a variabilidade das tensões em relação à posição periférica da barra. Esta variação de tensões residuais ao longo da posição periférica no material está relacionada com as tensões aplicadas durante o processo de manufatura. A Tabela 1 mostra a análise química do material, aço ABNT 1045. Para as medições pelo método de hole-drilling foram utilizadas duas fresas de diâmetros diferentes 1,50mm com diâmetro do furo de 1,80mm e 0,80mm com diâmetro do furo de 1,10mm, figura 1 . A Figura 2 mostra a metalografia do aço AISI 1045 trefilado, com tamanho de grão 7, observa-se nesta metalografia o alongamento dos grãos no sentido de trefilação, nota-se também que não há diferenças significativas entre a deformação dos grãos em regiões próximas a superfície em relação a deformação dos grãos no núcleo. Observou-se nas demais metalografias, não presentes neste trabalho, que não houve diferenças entre as diversas etapas do processo no que se refere ao tamanho de grão, microestrutura apresentada e bandeamento.

Tabela 1: Composição Química das amostras.

\begin{tabular}{c|c|c|c|c|c|c|c}
\hline Elemento & $\mathrm{C}$ & $\mathrm{Si}$ & $\mathrm{Mn}$ & $\mathrm{Cr}$ & $\mathrm{Ni}$ & $\mathrm{Al}$ & $\mathrm{Fe}$ \\
\hline$\%$ & 0,43 & 0,21 & 0,73 & 0,19 & 0,19 & $<0,01$ & 98,05 \\
\hline
\end{tabular}




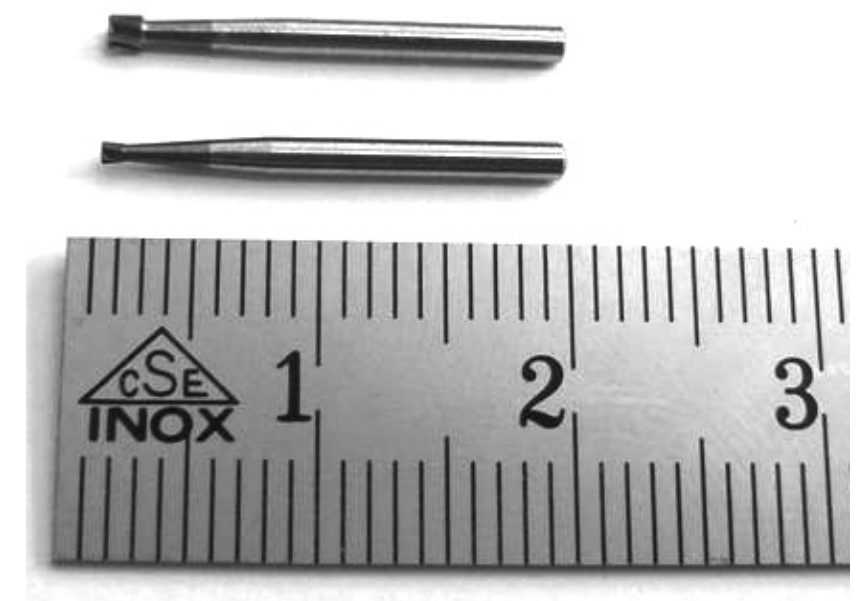

Figura 1: Comparação entre dois diferentes tamanhos de fresas utilizados nos ensaios de hole-drilling.

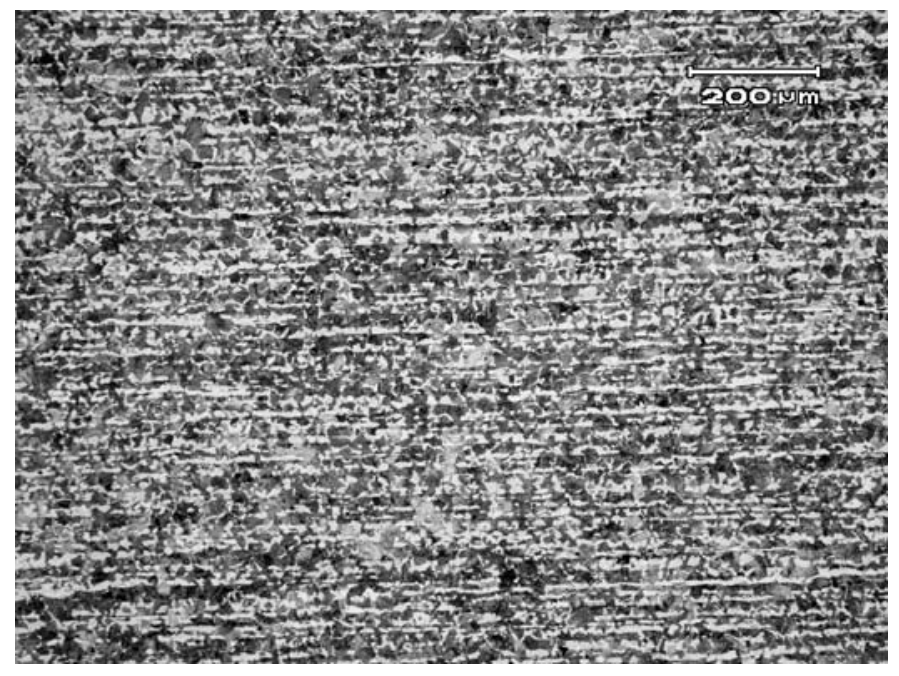

Figura 2: Metalografia do aço 1045, tamanho de grão 7, ataque Nital 2\%, aumento 50X.

Na retirada de amostras considerou-se a posição da barra em relação ao maquinário, desta forma, marcou-se nas amostras o sentido e a posição periférica conforme mostrado na Figura 2. As amostras foram analisadas por meio de difração de raios X (XRD) e método do furo cego (hole-drilling) para caracterizar a distribuição de tensões residuais na superfície em quatro posições da periferia conforme orientação apresentada na Figura 1, com medições para as posições $0^{\circ}, 90^{\circ}, 180^{\circ}$ e $270^{\circ}$, assim as mesmas posições periféricas analisadas na técnica de XRD foram medidas pela técnica de hole-drilling. Após análise dos valores superficiais, foram feitas medições através do método de difração de raios-x com remoção de camadas e do método do furo a fim de avaliar a variação das tensões com a profundidade, escolhendo-se para esta análise algumas posições consideradas críticas de acordo com os resultados superficiais.

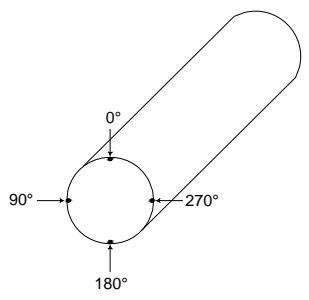

Figura 3: Sistema de orientação das amostras. 
O desenvolvimento de um sistema de tensões internas como resultado de deformações plásticas não homogêneas pode ser demonstrado com o exemplo de um endireitamento plástico de uma barra ( $1^{\mathrm{a}}$ etapa do processo de manufatura). A Figura 4(a) [2] representa a curvatura de uma barra, assume-se que esta barra é composta de várias finas camadas ao longo do raio desta barra. A tensão máxima trativa está na superfície superior desta barra e decai para um valor zero na posição referente à linha neutra, por outro lado esta tensão torna-se compressiva após a linha neutra atingir valores compressivos máximos na posição superficial oposta da barra. Se a tensão não exceder o limite de elasticidade do material em nenhum ponto, a remoção da tensão e momentos aplicados no material permitirá que o material volte ao estado original. Se, entretanto, a tensão máxima aplicada ao material exceder o escoamento, as camadas irão estender ou comprimir. Esta magnitude é maior na superfície e decai a zero na linha neutra do material.

No momento em que a tensão de endireitamento está sendo aplicada, a distribuição de tensões ao longo da largura da barra é representada na Figura 4(b), com as camadas 2 a 5 no regime plástico e a camada 1 no regime elástico da curva tensão deformação. Se o momento aplicado para o endireitamento é liberado a barra voltará para o seu estado original, mas não completamente, pois isto iria requerer uma completa reversão das tensões plásticas e elásticas aplicadas. O retorno elástico será diferente ao longo do raio da barra devido às diferenças de deformações plásticas impostas ao longo do raio da barra. Supondo que a camada 5 é isolada da camada 4, o descarregamento do ponto 5 na verdade fará com que este descarregamento só seja efetivo a partir da deformação plástica da camada 4, ou ainda, a camada 4 resistirá á deformação plástica da camada 5. Como resultado a camada 5 estará em compressão, as tensões de compressão na camada 5 serão $\Delta \varepsilon E$, onde $\Delta \varepsilon$ é a deformação residual mostrada na Figura 2(b) onde $\mathrm{E}$ é o modulo de Young do material. Para a camada 4 a situação é um pouco mais complicada, como a camada 3 irá resistir a extensão da camada 4, mas a camada 5 promove a extensão. A combinação resultante irá produzir uma tensão residual compressiva. É previsível afirmar que em algum ponto do material estes dois fatores encontrarão um equilíbrio resultando em tensões residuais nulas, como mostrado na camada 3. Para as camadas abaixo (2-1) o processo inicia-se novamente, sendo análogo para as demais camadas.

A distribuição de tensões residuais é apresentada na Figura 4(c). Dois pontos importantes podem ser notados na Figura 2(c): primeiro, há um equilíbrio das tensões trativas e compressivas; e segundo, a superfície que originalmente foi plasticamente deformada apresenta tensões residuais compressivas, pelo efeito da camada inferior estar tracionando a superfície do material. Além dos momentos aplicados pelos rolos no endireitamento ainda há o efeito superficial de contato entre o rolo e o fio-máquina, e esta pequena deformação superficial também irá induzir tensões no material [2].

O princípio básico do jateamento ( $2^{\mathrm{a}}$ etapa do processo de manufatura) é mostrado na Figura 4 [3, 4]. As granalhas são arremessadas contra a superfície do fio-máquina em alta velocidade e assim a superfície do fio-máquina é limpa e uma pequena porção superficial do material é deformada plasticamente.

De acordo com os estudos de $[\underline{4}, \underline{5}, \underline{6}]$ as deformações superficiais no jateamento induzem tensões residuais compressivas na superfície, pois o material usado no jateamento tem uma granulometria muito alta ocasionando pequenas deformações plásticas no material a nível macroscópico na superfície do material, as deformações impostas pelas granalhas induzem tensões trativas no núcleo do material e a superfície, desta forma, estará com tensões residuais compressivas conforme esquematizado na Figura 5.

Durante a trefilação ( $2^{\text {a }}$ etapa do processo de manufatura), as tensões residuais dependem fundamentalmente da distribuição inicial dos grãos - tamanho médio e dispersão - taxa de deformação ângulo de ferramenta - atrito entre ferramenta e o fio-máquina e do percentual de redução. Para passes de redução menores que $1 \%$, as tensões residuais longitudinais e circunferenciais são compressivas na superfície do material e trativas no núcleo. Segundo Dieter [7] as tensões residuais radiais são trativas no eixo chegando à zero na superfície. Industrialmente as reduções são mais elevadas, sendo comumente encontrados valores de redução entre 10-20\%. Neste caso o comportamento muda ficando o inverso do comportamento anterior, as tensões longitudinais e circunferenciais são trativas na superfície e as tensões residuais radias são compressivas no eixo.

O polimento e endireitamento por rolos cruzados - "PERC” ( $3^{\mathrm{a}}$ etapa do processo) é a última etapa do processo de manufatura, Figura 6. Esse equipamento é composto por dois rolos um rolo côncavo e outro convexo com um ângulo entre eles denominado $\beta$, este ângulo pode variar de $10-20^{\circ}$ dependendo das propriedades do material a ser endireitado e polido [] $]$. As barras cilíndricas ao passar por estes rolos de endireitamento e polimento sofrem uma pequena deformação superficial, induzida pelo rolo convexo, que induz tensões residuais compressivas em sua superfície, e este nível de tensões residuais compressivas induzidos pode mudar o comportamento do nível de tensões residuais presentes nas barras trefiladas, etapa anterior do processo de manufatura. Desta forma o comportamento passa de trativo na superfície para compressivo dependendo do ângulo com que estão posicionados esses rolos. 
(a)

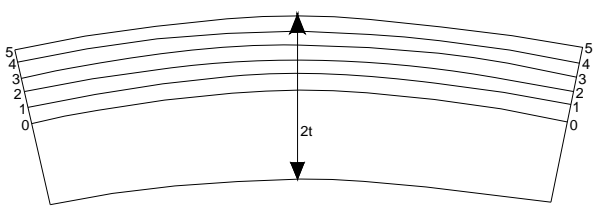

(b)

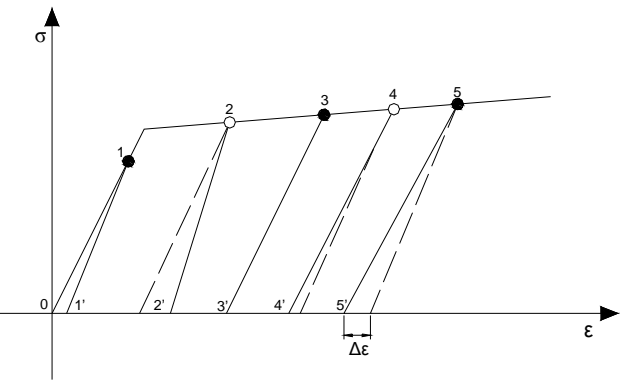

(c)

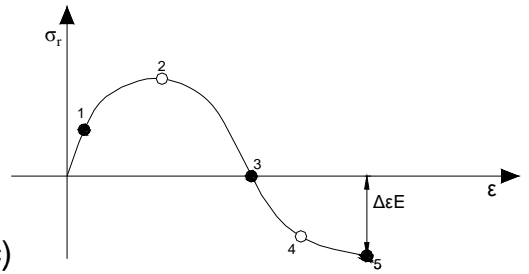

Figura 4: Geração de tensões residuais durante o endireitamento de um cilindro.

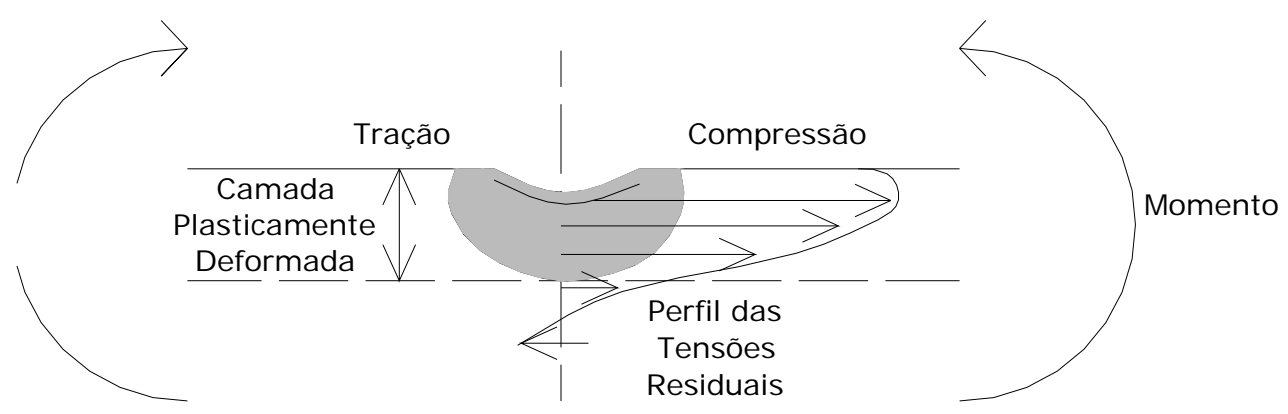

Figura 5: Diagrama esquemático do perfil de tensões residuais em uma simples indentação causada pelo jateamento.

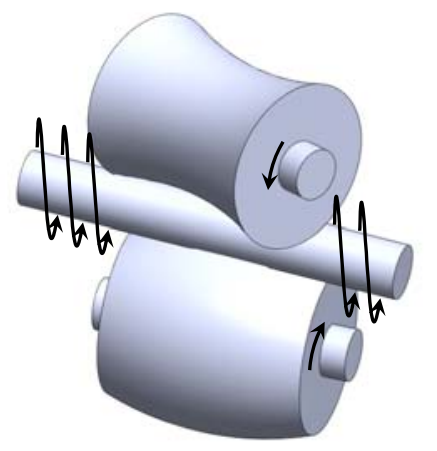

Vista trimétrica

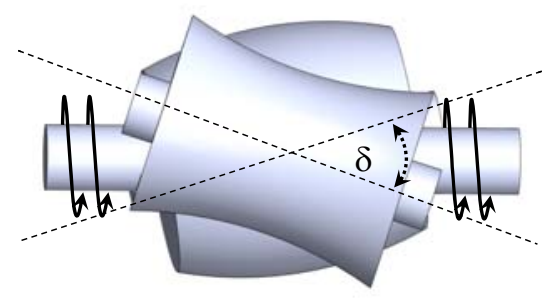

Vista superior

Figura 6: Desenho esquemático dos rolos cruzados de endireitamento e polimento de barras cilíndricas (“PERC”). 


\subsection{Medição de tensões residuais pelo método do furo cego - "hole-drilling"}

O método de análise de tensões residuais foi feito a partir da técnica de hole-drilling e também por difração de raios X (XRD). O método do hole-drilling baseia-se na deformação aliviada devido à introdução de um furo de pequenas dimensões na superfície do componente. O método de aquisição deste alívio de tensões é por "strain-gages”, utilizando para tanto extensômetros de resistência elétrica conforme a norma ASTM E-837 [9]. O método do furo cego é uma das mais utilizadas na determinação de tensões residuais superficiais, pois apresenta relativo baixo custo, mobilidade do equipamento, e possibilidade de aplicação a uma vasta variedade de materiais [1]. Por outro lado, o método do furo-cego apresenta algumas limitações, o método é considerado semi-destrutivo, pois dependendo do tipo de componente a ser analisado o furo pode causar um dano no mesmo $[\underline{1,} \underline{10}]$. Existem restrições na aplicação da técnica do furo-cego, uma das restrições refere-se à magnitude de tensões máximas a serem medidas podendo ser no máximo 30\% da tensão de escoamento do material $(\sigma y)$. Um material que apresenta tensões residuais superiores a 0,3бy ao ser submetido ao método do furo-cego poderá apresentar uma plastificação na borda do furo, esta, provocada pela redistribuição no estado de tensões na região onde houve a remoção de material e desta forma os resultados obtidos no ensaio não serão corretos podendo superar a tensão de escoamento do material [11], [10]. Neste caso, os resultados obtidos pela técnica tradicional do furo cego não são confiáveis, pois o algoritmo de cálculo das tensões é baseado em teorias linear-elásticas [11]. Alguns pesquisadores já quantificaram estes erros, podendo chegar a mais de $100 \%$ da tensão residual real apresentada pelo componente $[\underline{9}, \underline{10}, \underline{11}]$.

Segundo Kockelmann [12] para o cálculo das tensões residuais é necessário medir-se as deformações em três direções diferentes e conhecidas, atuando no mesmo plano. Assim, pode ser provado analiticamente que as tensões residuais principais apresentam a relação com a deformação radial medida nos três extensômetros descrita pela equação 1 , onde o $\sigma_{\max }$ e $\sigma_{\min }$ são as tensões residuais principais e $\varepsilon$ são as deformações relativas e $\bar{A}$ e $\bar{B}$ são as constantes de calibração [12].

$$
\frac{\sigma_{\max }}{\sigma_{\min }}=\frac{\varepsilon_{3}-\varepsilon_{1}}{4 \bar{A}} \pm \sqrt{\frac{\left(\varepsilon_{3}-\varepsilon_{1}\right)^{2}+\left(\varepsilon_{3}+\varepsilon_{1}-2 \varepsilon_{2}\right)^{2}}{4 \bar{B}}}
$$

O algoritmo para a determinação das tensões residuais é apresentado na equação 2, segundo Kockelmann e as direções das tensões residuais máximas principais são apresentadas na equação 3.

$$
\begin{aligned}
& \frac{\sigma_{\max }(\xi)}{\sigma_{\min }(\xi)}=\frac{\sigma_{a}(\xi)+\sigma_{b}(\xi)}{2} \pm \frac{1}{\sqrt{2}} \sqrt{\left(\sigma_{a}(\xi)-\sigma_{b}(\xi)\right)^{2}+\left(\sigma_{c}(\xi)-\sigma_{b}(\xi)\right)^{2}} \\
& \beta(\xi)=\frac{1}{2} \arctan \frac{2 \sigma_{b}(\xi)-\sigma_{a}(\xi)-\sigma_{c}(\xi)}{\sigma_{a}(\xi)-\sigma_{c}(\xi)}
\end{aligned}
$$

O método de Kockelmann apresenta vantagens em relação aos métodos convencionais, tais como o método da integral em relação a erros de instrumentação e medição, sendo menos sensível a estes erros e apresentando assim dados mais confiáveis [12].

\subsection{Medição de tensões residuais por difração de raios $X$}

Outro método de medição de tensões residuais largamente utilizado é a difração de raios X. Esta técnica é baseada no princípio de que quando um material é irradiado por raios X monocromáticos, para um cristal perfeitamente alinhado, no qual os átomos são empacotados regularmente e a distância entre os planos cristalográficos é definida pelas características físicas do material. A tensão medida no retículo cristalino e as tensões residuais associadas são determinadas a partir de constantes elásticas assumindo uma distorção linear do retículo cristalino. A medida das tensões residuais pelo método de raios X é feita a partir da contribuição de vários grãos do cristal e esta medida depende fundamentalmente da geometria de feixe que irá incidir na amostra bem como do tamanho de grão da amostra [13]. De acordo com a lei de Bragg [14] a profundidade de penetração das ondas de comprimento $\lambda$ na subsuperfície do material da amostra depende do ânodo usado, do tipo de material analisado e do ângulo de incidência do feixe na amostra e esta medida é sempre próxima à superfície da amostra. 


\section{$\lambda=2 d \operatorname{sen} \theta$}

A difração de raios $\mathrm{X}$ pode ser usada para medir com precisão os espaçamentos do retículo cristalino utilizando a lei de Bragg (equação 4) $[13,14]$, onde, $\lambda$ é o comprimento de onda da radiação usada, $\theta$ é a metade do ângulo de espalhamento, e "d" é o espaçamento interplanar médio para uma dada reflexão de um material cristalino, conforme exemplificado na Figura 7 [3]].

Em um material com tensões residuais, o espaçamento pode ser usado como um strain-gage, dando a medida da deformação na direção do vetor de difração. Se $\mathrm{d}_{0}$ são os espaçamentos do ângulo de Bragg medido para um material livre de tensões residuais, e tendo conhecimento prévio deste parâmetro, as tensões podem ser calculadas utilizando-se a equação 5 .

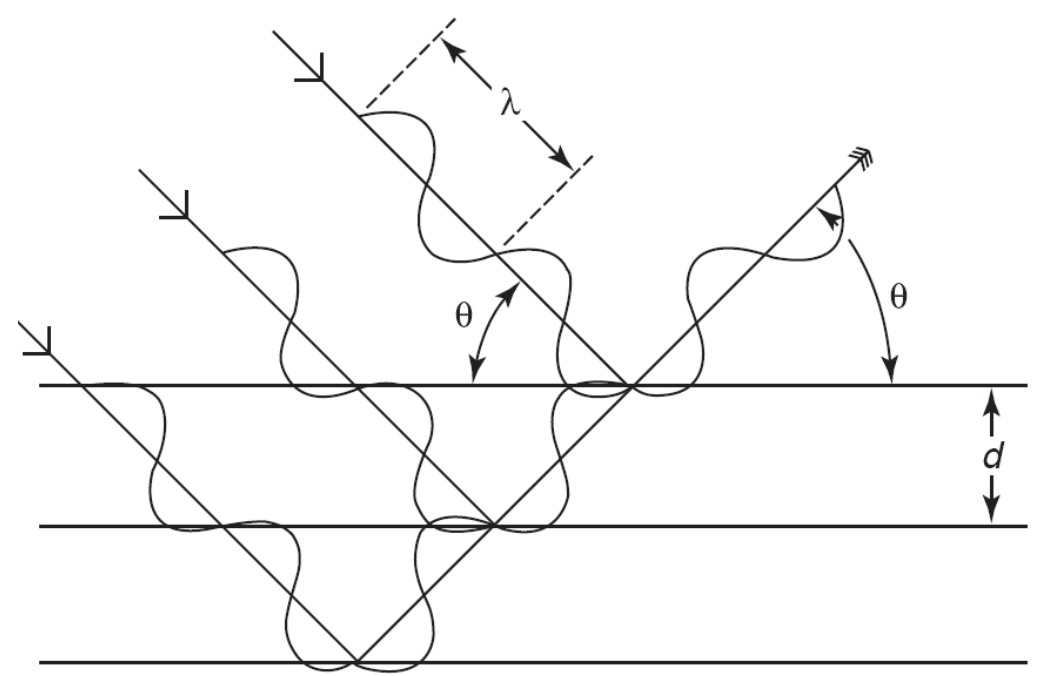

Figura 7: Espalhamento e difração de raios X em um arranjo cristalino.

$$
\varepsilon_{\phi \psi}=\frac{d_{\phi \psi}-d_{0}}{d_{0}}
$$

Segundo Fitzpatrick [3, 13], o estado de tensões em uma região nano, micro ou milimétrica do material é o tensor de segunda ordem representado pelos componentes de tensão normais ao sistema de coordenadas dado. Pode-se determinar o estado de tensões no material usando difração utilizando as regras de como o componente de tensão de segunda ordem transforma-se com a direção. Tendo as componentes de tensão do material, as tensões residuais podem ser calculadas usando-se a lei de Hooke, assim:

$$
d_{\phi \psi}=\left[\left(\frac{1+v}{E}\right)_{(h k l)} \sigma_{\phi} d_{0} \operatorname{sen}^{2} \psi\right]-\left[\left(\frac{v}{E}\right)_{(h k l)} d_{0}\left(\sigma_{\max }-\sigma_{\min }\right)+d_{0}\right]
$$

As barras foram medidas utilizando-se difratômetros - $\Psi$ (modo de inclinação lateral) equipados com tubos de raios X de Cr- Ka com abertura primária de $1 \mathrm{~mm}$ de diâmetro. As linhas de difração \{211\} do ferro- $\alpha$ foram registradas para $11 \Psi$ ângulos de inclinação na faixa de $45^{\circ}<\Psi<-45^{\circ}$. As tensões residuais foram calculadas usando o método de $\sin ^{2} \Psi$ [14] com $E=210000 \mathrm{MPa}$ e $v=0,28$. Perfis de tensões residuais obtidos por difração de raios $\mathrm{X}$ com remoção de camadas foram realizadas após a superfície de amostra ser atacada eletroliticamente durante um tempo de 120 segundos com uma solução de $80 \% \mathrm{H}_{2} \mathrm{SO}_{4} \mathrm{e}$ $20 \% \mathrm{H}_{3} \mathrm{PO}_{4}$. 


\section{RESULTADOS}

As Figuras 8-11 apresentam as tensões residuais em função da profundidade medidas pelas técnicas de difração de raios X (XRD) e hole-drilling (H.D) para as barras cilíndricas de aço ABNT 1045. Os resultados apresentados na técnica de hole-drilling podem apresentar um erro de medida devido a fatores operacionais do ensaio que segundo Grant et al. [15] estão na ordem de $10 \%$. Considera-se este valor como sendo o valor mínimo de incerteza para a técnica de hole-drilling, levando-se em conta todos os fatores experimentais que levam a erros, tais como, manuseio da amostra, preparação da superfície, alinhamento do "strain-gage". A barra de erros nas medições por difração de raios X representa o desvio padrão calculado a partir de fatores experimentais do equipamento, este erro foi calculado utilizando-se o software Stress ${ }^{\circledR}$ versão 2.0.

Na Figura 8 é apresentada uma comparação entre as medições de tensões residuais obtidas pelo método de difração de raios X e hole-drilling para a barra trefilada utilizando-se um ângulo de ferramenta de $20^{\circ}$. Nesta Figura, para a técnica de hole-drilling utilizou-se uma fresa padrão $(1,50 \mathrm{~mm})$ com diâmetro do furo de $1,80 \mathrm{~mm}$.

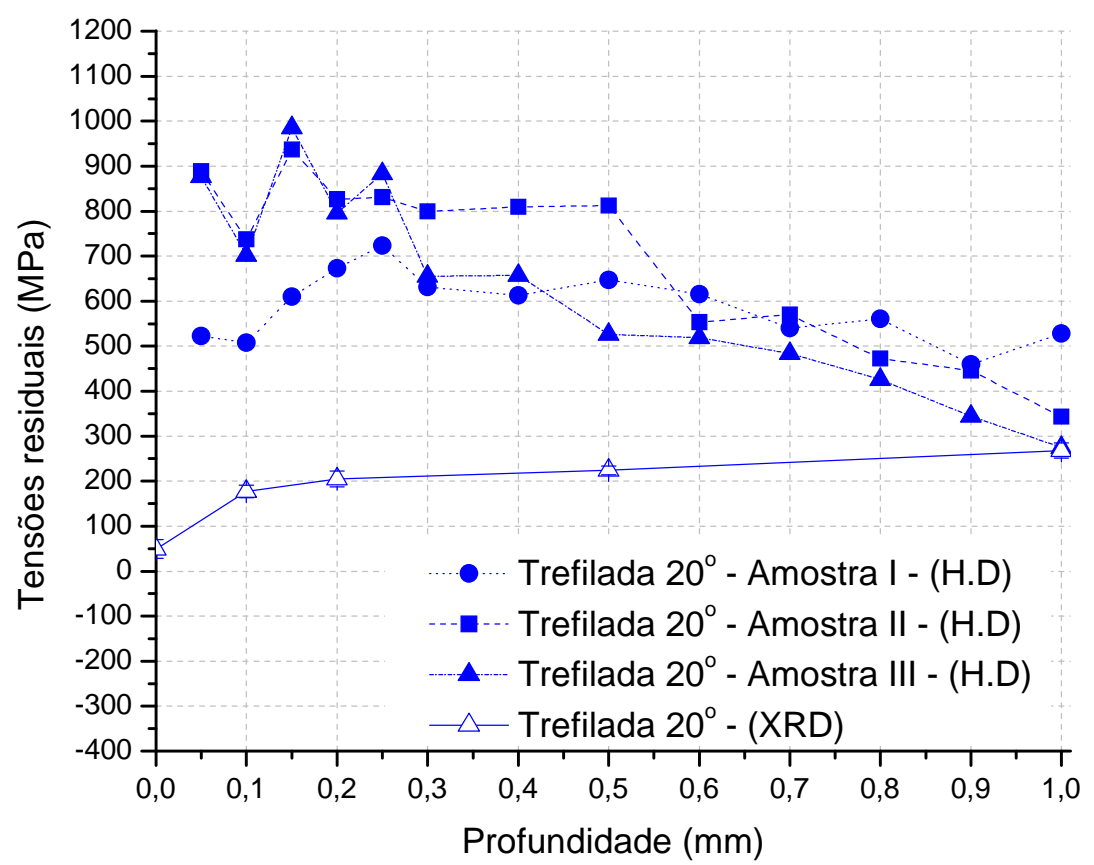

Figura 8: Comparação entre as tensões residuais medidas por hole-drilling (H.D) e difração de raios X (XRD) para a etapa de trefilação utilizando uma fresa convencional com 1,50mm de diâmetro.

A Figura 9 apresenta as tensões residuais em função da profundidade medidas pelas técnicas de difração e hole-drilling para as etapas de jateamento, trefilação e polimento e endireitamento por rolos cruzados ("PERC"). Na técnica de hole-drilling para as etapas de jateamento e endireitamento por rolos cruzados ("PERC") utilizou-se uma fresa padrão $(1,50 \mathrm{~mm})$ com diâmetro do furo de $1,80 \mathrm{~mm}$. Para a etapa de trefilação utilizou-se uma fresa de menor diâmetro $(0,80 \mathrm{~mm})$ com diâmetro do furo de $1,10 \mathrm{~mm}$. Objetivou-se a utilização da fresa de menor diâmetro para a etapa de trefilação devido aos resultados apresentados pelas técnicas de difração de raios X e hole-drilling mostrados na Figura 8 não estarem coerentes. A utilização de uma fresa de menor diâmetro evita a plastificação da borda do furo conforme também mostrado via elementos finitos por Focht [16]. Foch afirma em seus estudos que após uma zona de aproximadamente 1,9 vezes o diâmetro do furo os efeitos da plastificação sejam mínimos. Assim, acredita-se que com a utilização de uma fresa de menor diâmetro este efeito seja minimizado não sendo detectado pelo "strain-gage", resultando em medições reais, levando-se em conta somente os efeitos de tensões residuais e não os efeitos da plastificação, como encontrados utilizando-se uma fresa de tamanho convencional.

A Figura 10 apresenta uma comparação das medições realizadas entre as técnicas de difração de raios-X e hole-drilling para a etapa de trefilação utilizando um ângulo de ferramenta de $18^{\circ}$. Na técnica de hole-drilling utilizou-se uma fresa uma fresa de $0,80 \mathrm{~mm}$ de diâmetro com diâmetro do furo de $1,10 \mathrm{~mm}$.

A Figura 11 mostra as tensões residuais em função da profundidade utilizando-se as técnicas de difração de raios X e hole-drilling para a etapa de polimento e endireitamento por rolos cruzados. Para a 
realização do perfil de tensões residuais pelo método de hole-drilling utilizou-se a fresa padrão com diâmetro de 1,50mm, desta forma, o diâmetro do furo foi de $1,80 \mathrm{~mm}$.

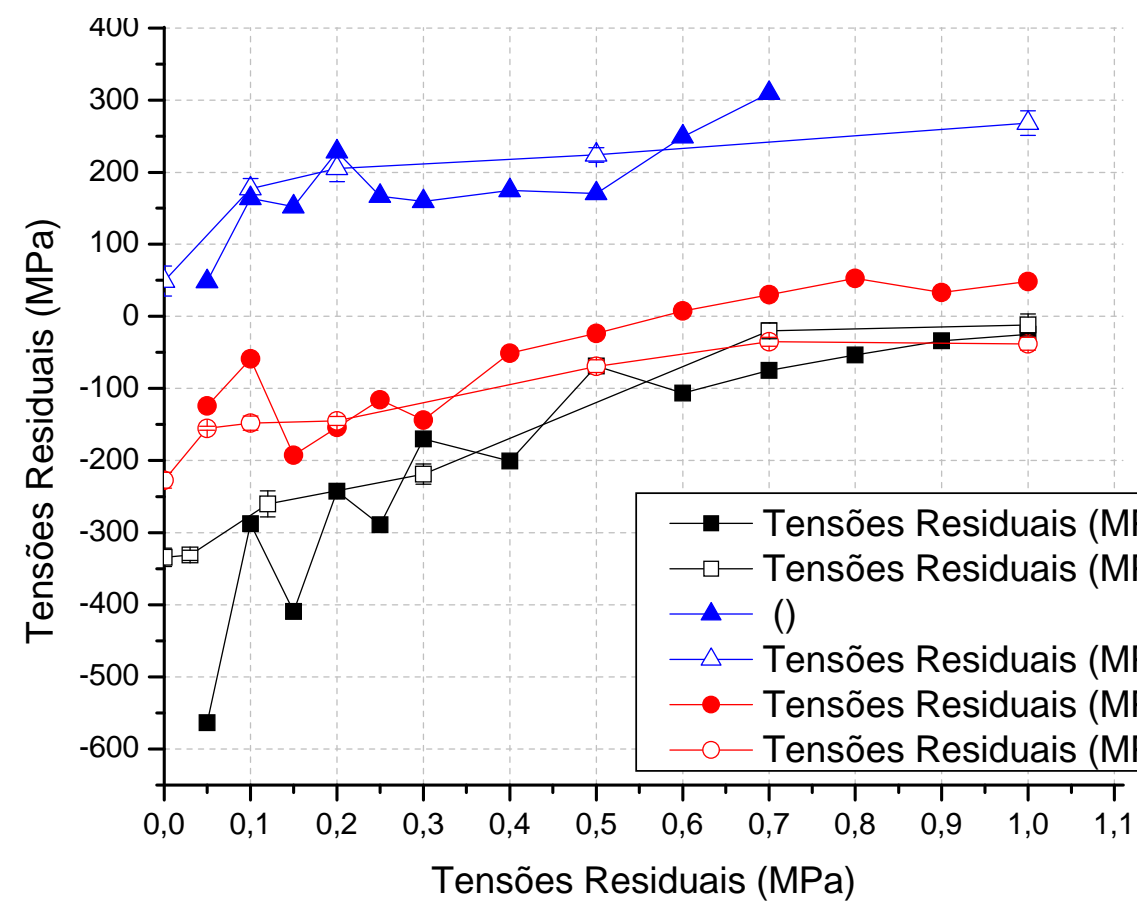

Figura 9: Comparação entre as tensões residuais medidas por hole-drilling (H.D) e difração de raios X (XRD) para a etapa de jateamento, trefilação e "PERC".

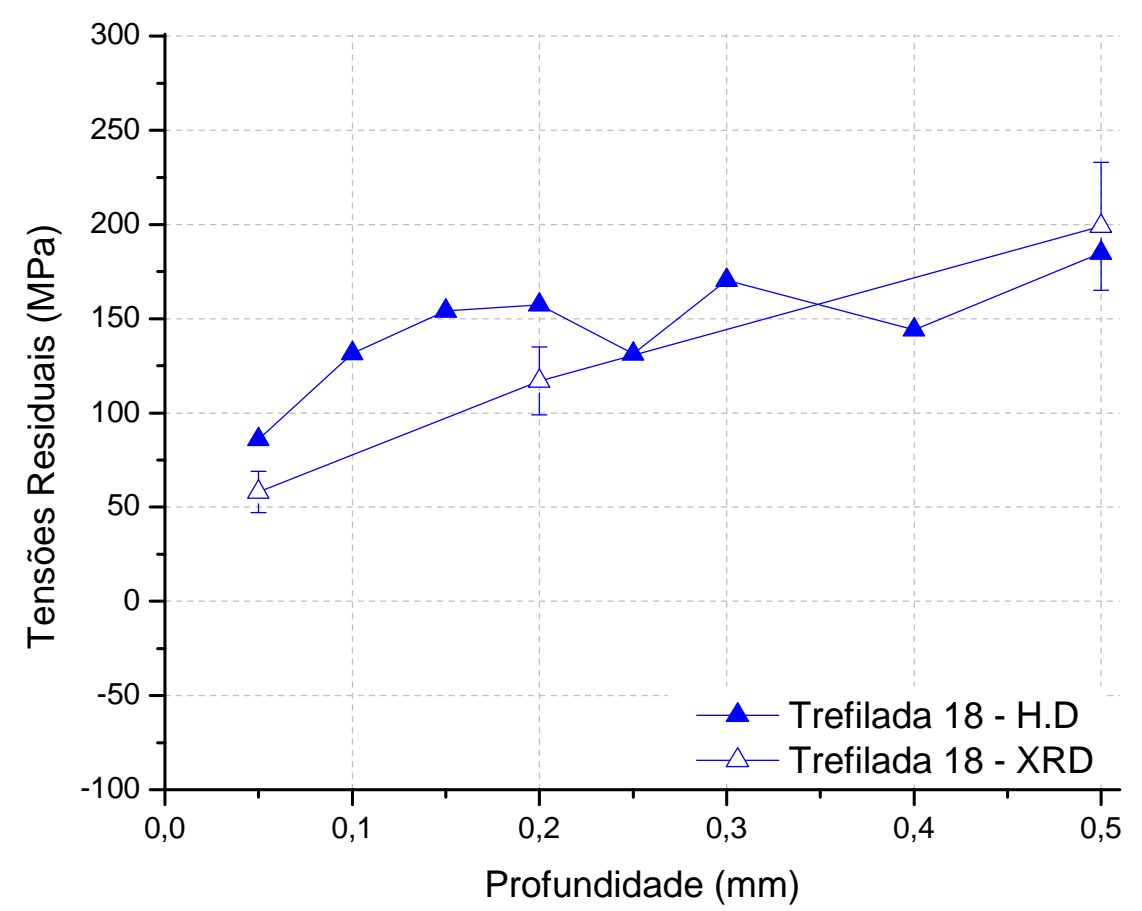

Figura 10: Comparação entre as tensões residuais medidas por hole-drilling e difração de raios $X$ para a etapa de trefilação, fresa de $0,80 \mathrm{~mm}$ de diâmetro. 


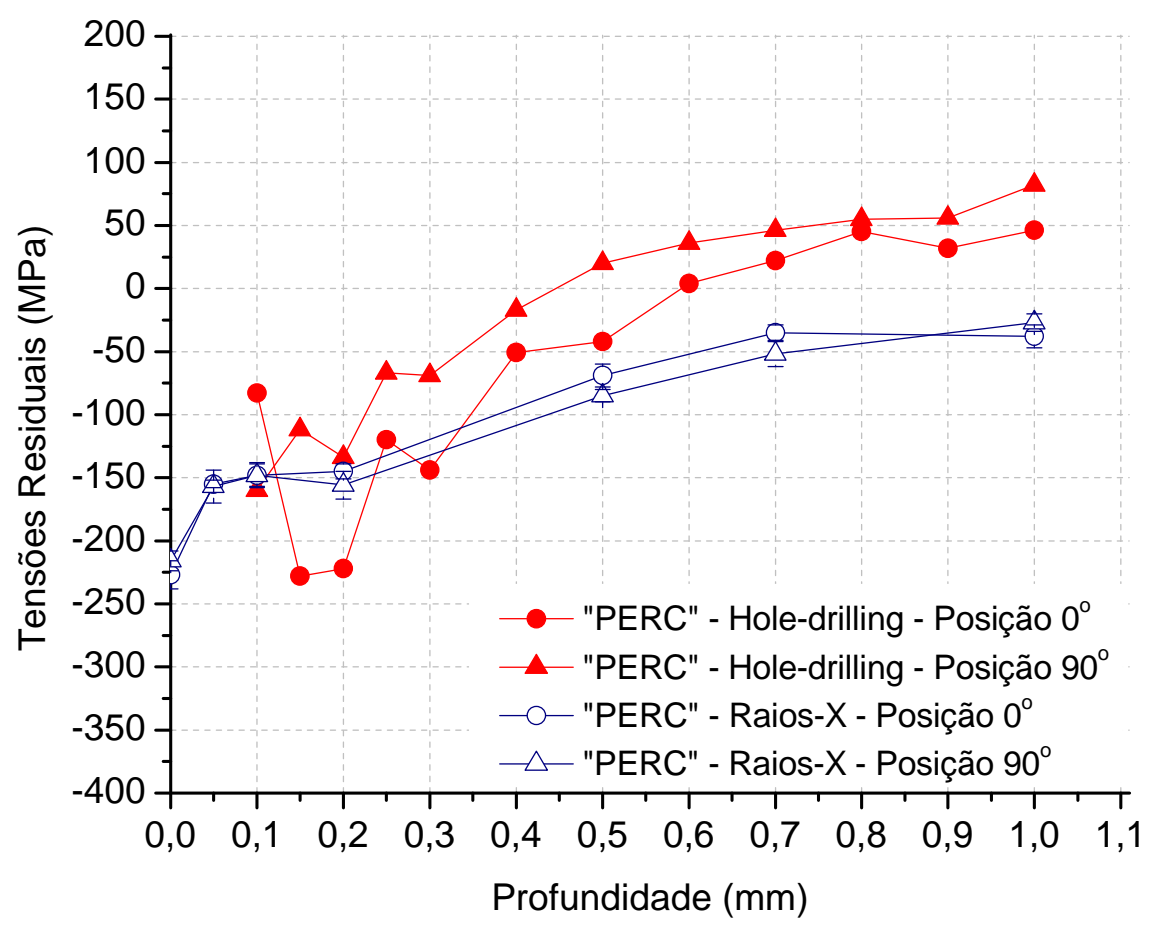

Figura 11: Comparação entre as tensões residuais medidas por hole-drilling e difração de raios X para a etapa de “PERC”.

\section{DISCUSSÃO}

Nos primeiros ensaios realizados não houve concordância entre o método de hole-drilling com os resultados observados na técnica de difração de raios $\mathrm{X}$, obtendo-se valores muito discrepantes conforme mostrado na Figura 5.

As tensões residuais longitudinais na barra trefilada medidas pelo método de hole-drilling e difração de raios $\mathrm{X}$ são apresentadas na Figura 8. As tensões residuais medidas pelo método de hole-drilling ultrapassaram o limite de escoamento do material que é próximo de 650MPa. Estas discrepâncias de resultados também já foram observadas por outros pesquisadores $[\underline{10}, \underline{11}, \underline{16}, \underline{17}, \underline{18}]$. Na Figura 12 são mostrados os resultados obtidos por Yoshihisa [18] em cordões de solda, observa-se que também houve diferenças de até 300MPa nas medições obtidas pelo método do furo cego (hole drilling) comparadas com as medições de difração de raios $X$.

Uma explicação para estas diferenças é que ao se fazer o furo para medição do nível de tensões residuais cria-se um novo estado de tensões no material fazendo-se com que a superfície próxima ao furo escoe plasticamente, ocasionando uma deformação plástica superficial na periferia do furo, e assim o método neste caso não apresenta resultados satisfatórios. Porém de acordo com a teoria formulada por Hampton \& Nelson [17] e Focht \& Schiffner [16] explicando os motivos desta incoerência de resultados, para resolver este problema este estudo propõe a utilização de uma fresa com diâmetro menor ao utilizado, eliminando desta forma os efeitos detectados pelo "strain gage” devido à deformação plástica superficial causada pelas tensões aliviadas pelo furo. Desta forma observaram-se valores satisfatórios de tensões residuais nas barras trefiladas, conforme exibidos nas Figuras 9 e 10 mostrando a coerência desta medida em relação à técnica de difração de raios $\mathrm{X}$.

Na Figura 9 observa-se o nível de tensões residuais longitudinais em função da profundidade, apresentado nas etapas de pré-endireitamento, jateamento e trefilação medidos pelo método do furo cego e difração de raios X. A etapa de trefilação induz tensões residuais trativas e este comportamento mantendo um nível próximo de 200MPa com o aumento da profundidade até $1 \mathrm{~mm}$. Este comportamento foi observado pelas duas técnicas de medição, porém para a técnica de hole-drilling foi necessária a substituição da fresa para uma de menor diâmetro, como comentado anteriormente. Os resultados apresentados nas técnicas de difração de raios $\mathrm{X}$ e hole-drilling são idênticos, da mesma forma que os apresentados na etapa de jateamento. No polimento e endireitamento por rolos cruzados ("PERC”), as tensões trativas superficiais impostas pela trefilação são superadas pelas tensões aplicadas no polimento e endireitamento por rolos cruzados ("PERC”) e tornam-se compressivas novamente na superfície. Os resultados observados nas técnicas de hole-drilling e difração de raios X são similares em termos de comportamento e níveis de tensões. 

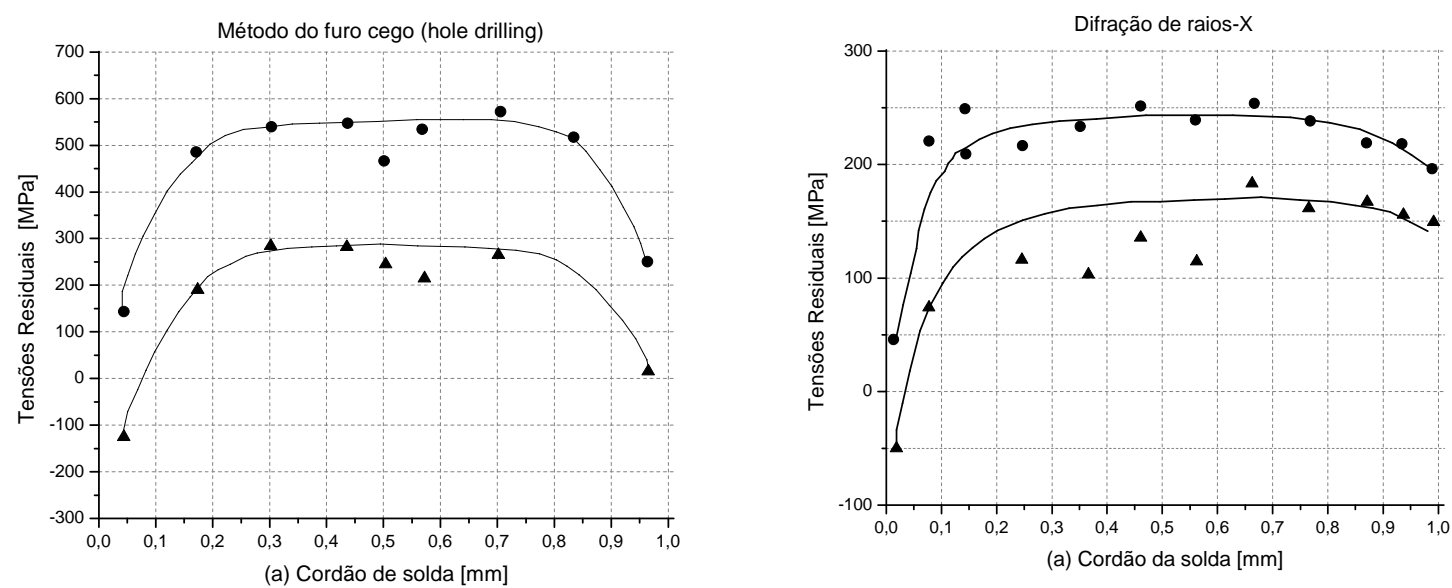

Figura 12: Comparação entre as tensões residuais em juntas soldadas medidas por hole-drilling e difração de raios X obtidos por Yoshihisa [18].

Na Figura 10 observa-se a comparação de tensões residuais longitudinais medidas pelas técnicas de difração de raios X e hole-drilling para a barra polida e endireitada por rolos cruzados (“PERC”). Nota-se a coerência de resultados medidos nas duas técnicas, as tensões residuais apresentam um comportamento compressivo em profundidades próximas à superfície e tendem a valores menos compressivos a partir de 0,5 mm medidos pela técnica de difração de raios X e trativos para as medições via hole-drilling para esta mesma profundidade. Apesar da diferença de comportamento das tensões após 0,5mm os resultados obtidos nas duas técnicas são bem próximos. Além disso, estas diferenças podem ser explicadas pela variabilidade durante o processo de manufatura das barras cilíndricas.

Na Figura 11 observa-se uma comparação do perfil de tensões residuais longitudinais obtidos via difração de raios X com remoção de camadas e o método de hole-drilling realizado com uma fresa de menor diâmetro, sendo o diâmetro do furo $1,10 \mathrm{~mm}$ para a fresa de menor diâmetro $(0,80 \mathrm{~mm})$ e $1,80 \mathrm{~mm}$ para a fresa convencional (1,50mm). A partir dos resultados obtidos e a comparação dos ensaios com o método de difração de raios X observou-se resultados satisfatórios e confiáveis. De acordo com Grant et al [15] e Vihsay [19] a única desvantagem de se usar uma fresa de diâmetro menor é a profundidade de validade dos resultados obtidos no ensaio ser menor $0,7 \mathrm{~mm}$ comparando-se com o diâmetro padrão que é de $1 \mathrm{~mm}$.

\section{CONCLUSÕES}

A partir dos resultados obtidos pode-se observar a concordância entre as técnicas de medição de tensões residuais compressivas em barras cilíndricas. Entretanto, não houve concordância de resultados de medição de tensões residuais trativas utilizando-se os métodos convencionais de medida. Para que houvesse concordância nos resultados para tensões residuais trativas foi necessária a utilização de uma fresa de menor diâmetro no método de hole-drilling. Desta forma, houve resultados coerentes para as duas técnicas.

\section{AGRADECIMENTOS}

Os autores agradecem a FINEP, CAPES e DFG pelo suporte financeiro para a realização deste trabalho e o IWT- Stiftung Institut für Werkstofftechnik - Universidade de Bremen - Alemanha pela parceria na realização das medições de tensões residuais por difração de raios X.

\section{BIBLIOGRAFIA}

[1] LU, J., Handbook of Measurement of Residual Stresses, 1 ed., Senlis, France, Society for Experimental Mechanics - Inc., 1996.

[2] WANG, Z., "Residual stress in the forming of materials”, In: ASM Handbook of residual stress and deformation of steel, American Society for Metals International, pp. 141-147, 2002. 
[3] FITZPATRICK, M.E., LODINI, A., Analysis of Residual Stress by Diffraction using Neutron and Synchrotron Radiation, 1 ed., London, Taylor \& Francis, 2003.

[4] TANGE, A., ANDO, K., "Study on shot peening processes of coil springs", In: Proceedings of the 6th International Conference on Residual Stresses, pp. 897-904, Oxford, United Kingdom, 2000.

[5] TOWNSEND, D.P., ZARETSKY, V., "Effect of shot peening on the surface fatigue life of carburized and hardened AISI 9310 spur gears”, NASA Technical Paper 2047, 1982.

[6] MURPHY, M., “Blasting and shot peening”, Metal Finishing, vol. 95, Issue 2, pp. 16-18 Feb. 1997.

[7] DIETER, G.E., Metalurgia Mecânica, 2 ed., Rio de Janeiro, Guanabara Dois, 1986.

[8] WU, B.J., CHAN, L.C., LEE, T.C., AO, L.W., “A study on the precision modeling of the bars produced in two cross-roll straightening”, Journal of Materials Processing Technology, 99, pp. 202-206, 2000.

[9] ASTM E-837, "Standard test method for determining residual stresses by the hole-drilling strain-gage method”, Annual Book of ASTM Standards, v. 3, n. 1, American Society for Testing of Materials, 2002.

[10] SOARES, A.C.C., Uma nova metodologia numérica para avaliar os erros gerados na medição de tensões residuais pelo método do furo cego em presença de tensões elevadas, Tese de D.Sc., PUCRio, Rio de Janeiro, 2003

[11] ERMINI M., Plasticity effects in residual stress measurement by the hole drilling method, Strain, v.36, Issue 2, pp. 55-59, 2000.

[12] KOCKELMANN, H. E., SCHWARZ, T., "Die Bohrlochmethode-ein für viele Anwendungsbereiche optimales Verfahrenzur experimentellen Ermittlung von Eigenspannungen”, Messtechnische Briefe von HBM, 29(2), pp. 33-38, 1993.

[13] FITZPATRICK, M.E., FRY, A.T., Measurement Good Practice Guide No. 52, Determination of Residual Stresses by X-ray Diffraction - Issue 2, National Physical Laboratory - NPL, United Kingdom, 2005.

[14] ZOLOTOREVSKY, N. YU., KRIVONOSOVA, N. YU., "Effect of ferrite crystals plastic anisotropy on residual stresses in cold-drawn steel wire”, Materials Science and Engineering, A205 pp. 239-246, 1996.

[15] GRANT, P.V., LORD, J.D., An evaluation of four hole drilling analysis techniques with respect to nonuniform residual stress fields, National Physical Laboratory, United Kingdom, August, 2002.

[16] FOCHT, G., SCHIFFNER, K., "Determination of residual stresses by an optical correlative hole-drilling method”, Experimental Mechanics, v. 43, Issue 1, pp 97-104, 2003.

[17] HAMPTON, R.W., NELSON, D.V., "Comparison of residual stress measurements in welded thin plates by x-Ray and hole drilling methods”, American Society for Mechanical Engineers - Non destructive Evaluation, v. 5, pp. 171-176, 1989.

[18] YOSHIHISA, E., KITSUNAI, Y., MAEDA, Y., "Residual stress measurements of welded plates by xray and hole drilling methods”, In: Residual Stresses III: Science and Technology, 1ed., Taylor \& Francis, 1992.

[19] VISHAY, http://www.vishay.com. Acessado em Setembro de 2008. 